\title{
Cortisol levels and serum antioxidant status following chemotherapy
}

\author{
Eugenia Limberaki ${ }^{1}$, Phaedra Eleftheriou ${ }^{1}$, Georgios Gasparis ${ }^{1}$, Eugenios Karalekos ${ }^{1}$, Vassilis \\ Kostoglou ${ }^{2^{*}}$, Christos Petrou ${ }^{1}$ \\ ${ }^{1}$ Department of Medical Laboratory Studies, Alexander Technological Educational Institute of Thessaloniki, Thessaloniki, Greece; \\ ${ }^{2}$ Department of Informatics, Alexander Technological Educational Institute of Thessaloniki, Thessaloniki, Greece; \\ *Corresponding Author: vkostogl@,it.teithe.gr
}

Received 28 April 2011; revised 1 July 2011; accepted 13 July 2011.

\begin{abstract}
Chemotherapy results in increased free radical formation and depletion of tissue antioxidants. Moreover, patients receiving chemotherapy are under emotional stress, which is also accompanied by low antioxidant levels. In the present study, we measured cortisol, the main stress hormone, and total antioxidant capacity (TAC) in serum of 51 cancer patients during chemotherapy. Antioxidant activity was estimated by measuring the influence of serum in oxidation of ABTS (2,2'-azino-bis (3-ethylbenz-thiazoline6-sulfonic acid to ABTS+ by methmyoglobin (Antioxidant kit of Cayman). Serum cortisol was measured using an ELISA colorimetric assay. Serum TAC was significantly decreased $(75 \%$ decrease compared to normal levels, $p=0.001$ ) in all patients during chemotherapy, while blood cortisol concentration was increased by $10 \%$, (p $=0.044)$. Lower antioxidant levels and higher cortisol concentration were detected in patients receiving chemotherapeutic drugs daily, compared to the ones receiving chemotherapy once a week. A difference between sexes was observed with male patients presenting lower antioxidant status and higher cortisol levels than females. A significant and persistent decrease in antioxidant capacity accompanied by increased cortisol concentration was observed in all patients during chemotherapy. This fact, which is probably generated by biological and emotional stress, increases the probability of harmful side effects and organism weakening and needs to be considered during patients' treatment.
\end{abstract}

Keywords: Antioxidant Capacity; Cortisol; Chemotherapy; Cancer

\section{INTRODUCTION}

Cancer cells themselves seem to cause a decrease in antioxidant status [1]. Chemotherapy acts by killing cells that divide rapidly, one of the main properties of most cancer cells. Additionally, the action of most chemotherapeutic agents is based in creating reactive oxygen species (ROS) [2,3], and these agents are associated with depletion of plasma and tissue antioxidants [4-7]. ROS are cytotoxic molecules and key mediators in signaling cascades. Increased plasma lipid peroxidation and thiobarbituric acid-reactive species accompany oxidative stress induced in patients receiving chemotherapy $[8,9]$. Glutathione (GSH) concentrations are markedly reduced by chemotherapeutic agents, such as busulfan, cermustine (BCNU), and cisplatin [10]. Cisplatin is a platinum containing anti-cancer drug reacting in vivo and causing cross linking of DNA, which triggers to apoptosis. In addition, apoptosis induced by cisplatin is inhibited by ROS scavengers, including N-acetyl cysteine, MnTBAP and $\mathrm{C}_{60}$. Cisplatin promotes ROS production, which in turn contributes to Fas receptor aggregation and cell death. Newer anticancer drugs like nutlins act directly against abnormal proteins in cancer cells; this is termed target therapy and is technically not chemotherapy. These drugs kill cancer cells via ROS-independent pathways. Phagocytosis of cancer cells may be another reason for the generation of free radicals during chemotherapy [11]. The low serum total antioxidant capacity observed in patients suffering from cancer reflects this imbalance [12]. Insufficient defense against free radicals may have harmful results, which may be the reason for serious undesired side effects of chemotherapy. Moreover, it is hypothesized that ROS may facilitate metastasis [13].

Many patients being treated for cancer receive dietary supplements, particularly antioxidants, during chemotherapy and radiotherapy $[14,15]$. Although some preclini- 
cal data show that antioxidants might reduce the effectiveness of chemotherapy [16], other studies report similar or better response in chemotherapy when combined to antioxidant-rich diet [17-19]. For example, while an excess in vitamin $\mathrm{C}$ is believed to facilitate glucose transportation into cancer cells, thus inducing proliferation [20-22], antioxidant vitamins such as C and E have been successfully used as supplements for ameliorating the side effects of chemotherapy $[23,24]$. In general, if generation of reactive oxygen species by a cancer chemotherapeutic agent plays a role in its cytotoxicity, antioxidants may interfere with the drug's antineoplastic activity. However, if the reactive species are responsible only for the drug's side effects, antioxidants may actually reduce the severity of such effects without interfering with the drug's antineoplastic activity $[2,25]$. As a consequence, different forms of antioxidant molecules have been categorized either as interfering or not interfering with anticancer therapy.

Cortisol, usually referred to as the "stress hormone", is involved in the organism's response to stress and anxiety. It triggers all metabolic mechanisms leading to production of compounds used as energy sources in emergency conditions. It increases glucose and free aminoacid concentration in serum by inducing gluconeogenesis, thus inhibiting protein synthesis and promoting hydrolysis of lipids and proteins, and mobilization of extra hepatic amino acids and ketone bodies. Cortisol levels are affected by the circadian cycle with highest values observed early in the morning. Increased cortisol concentrations are measured in blood serum of people under biological or emotional stress, depression, sleep deprivation, fever, hypoglycemia, anorexia nervosa and after surgery [26-28]. A 3\% - 10\% increase in cortisol levels has been observed in cancer patients varying according to the severity of the disease [29]. Cortisol is one of the main biomarkers of depression and is often used for monitoring depression therapies [30]. Moreover, increase in cortisol concentration has been associated with decrease in total antioxidant levels and low total antioxidant capacity (TAC) is a common feature in all conditions where elevated cortisol concentrations are observed [31]. The levels of some tissue antioxidants are inversely related to cortisol tissue levels [32]. Antioxidant depletion may result as a consequence of various pathways activated by cortisol or may be the effect of free radical production during cortisol metabolism, since oxidative metabolism of cateholamines produces cinones which react with superoxide anion, $\mathrm{O}_{2}{ }^{\circ}$, and hydrogen dioxide, $\mathrm{HO}_{2}{ }^{\circ}$. Antidepressant therapy resulting in decrease of cortisol levels also results in increase of antioxidant concentration. Moreover, it has been stated that augmentation of antioxidant defense could serve as an important mechanism underlying the neuroprotective pharmacological effects of these drugs [33].

Cancer patients on chemotherapy are under strong biological and emotional stress. This may lead to high cortisol levels which may be an additional cause for the reduction of protective antioxidants and induction of undesired side effects.

In this study, we measured the total antioxidant capacity and cortisol levels in serum of 51 cancer patients receiving chemotherapeutic agents once a day or once a week and compared the concentrations of the two parameters between 1) patients and healthy people, 2) patients receiving the treatment once/day and once/week), and 3 ) patients of different sex.

\section{METHODS}

In the present study, the level of total blood antioxidants and cortisol levels were measured in blood serum of 51 (18 male and 33 female) Greek cancer patients on chemotherapy. All blood samples were collected before treatment between 8.00 and 9.00 a.m. All patients were volunteers, informed about the project, and did not follow any specific diet. The serum samples were stored for 3 - 15 days at $-80^{\circ} \mathrm{C}$ (Serum can be stored at $-80^{\circ} \mathrm{C}$ for 1 month as it is described in the Technical Bulletin of Antioxidant Assay Kit of Cayma). Lipemic and hemolysed samples-when found-were excluded. 19 of the patients received their chemotherapy agents once a day and 32 received their therapy once a week. Most chemotherapy agents consisted of busulfan, etoposide and cyclopjosphamide, or cytosine, diaziquone and thiotepa. For comparisons, samples from 32 men and women of about the same age with no known health problem were also collected and used as controls.

Evaluation of TAC was done using the antioxidant Assay Kit of Cayman. Serum cortisol was measured using the immunoenzyme assay kit of HUMAN. Absorbance rates were measured using START-FAX 2100 microtitre plate reader.

\subsection{Evaluation of Serum Total Antioxidant Capacity}

TAC measurement was carried out using the antioxidant Assay Kit of Cayman. Antioxidants inhibit the oxidation of ABTS (2.2'-azino-di 3-ethylbenthiazolinesulfonate) to ABTS+ by methmyoglobin. Trolox, the water soluble analogue of vitamin E, was used as control. Antioxidant concentration is quantified in millimolar Trolox equivalents [34].

\subsection{Evaluation of Serum Cortisol}

Serum cortisol was measured using the immunoen- 
zyme assay kit of HUMAN. The method is based on competitive interaction of serum cortisol and a cortisolenzyme conjugated provided by HUMAN for a limited number of monoclonal anti-cortisol antibodies bound to the wells of a microtitre ELISA plate. The amount of the bound hormone-enzyme conjugate is inversely proportional to the concentration of cortisol in the specimen. After removal of unbound conjugate by washing, a substrate solution is added and a blue color develops which changes to yellow by the addition of stop solution. The absorbance is measured at $450 \mathrm{~nm}$ using a mictotitre plate reader. Concentration of unknown specimens is interpolated from a dose response curve generated by utilizing serum calibrators of known cortisol concentration [35].

\subsection{Statistical Analysis}

Statistical analysis of data was carried out with SPSS [36]. The Student's $t$ test for independent samples was used to compare the blood samples of cancer patients with them of healthy people. As this type of data follow the normal distribution, we used the Student t-test to detect significant differences between groups.

\section{RESULTS}

Measurement of the TAC in blood serum of 51 cancer patients receiving chemotherapy and 32 healthy individuals of the same age revealed a $75 \%$ decrease in serum antioxidants of treated cancer patients $(p=0.001$ according to the Student's t-test). Measurement of cortisol levels in the same blood samples revealed a $10 \%$ increase in cortisol concentration in the patients' group $(\mathrm{p}=0.044)$ (Figure 1).

TAC decrease was greater in patients receiving chemotherapy once a day compared to the patients receiving chemotherapy once a week (Figure 2).

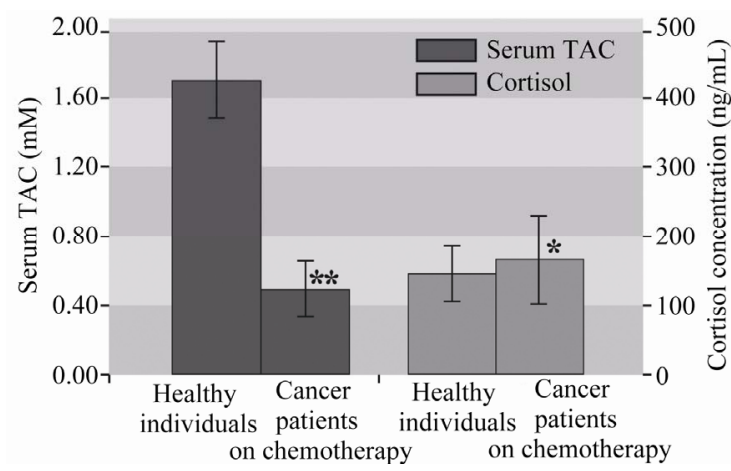

Figure 1. Total antioxidant capacity and cortisol levels in blood serum of cancer patients receiving chemotherapy $(n=51)$ and healthy individuals $(\mathrm{n}=32)$ of the same age (significance levels according to the Student's t-test: $* \mathrm{p}=0.044,{ }^{* *} \mathrm{p}=$ $0.001)$. The vertical bars represent standard deviation (SD).

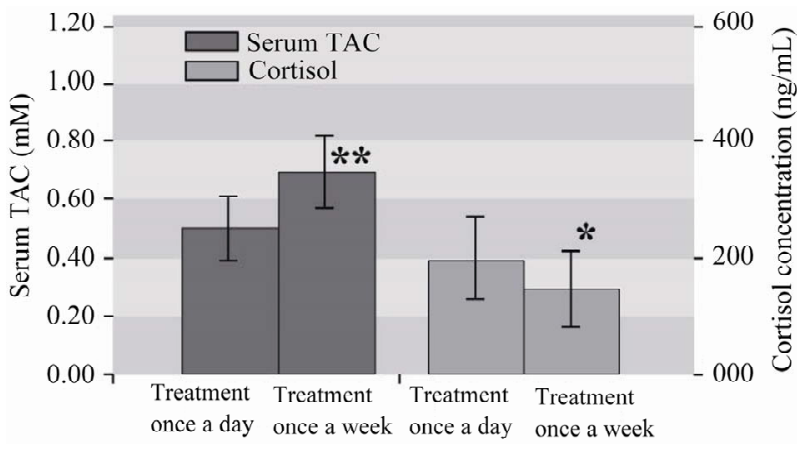

Figure 2. Total antioxidant capacity and cortisol levels in blood serum of cancer patients receiving chemotherapy once a day $(n=19)$ and once a week $(n=32)$ (significance levels according to the Student's t-test: $* p=0.101, * * p=0.001)$. The vertical bars represent SD.

A statistically significant difference $(p=0.001)$ was observed between the two groups of patients with total blood antioxidant concentration in daily receiving chemotherapy patients being 37\% lower compared to the weekly treated individuals. Greater elevation of cortisol levels was measured in daily treated patients (D-T P) compared to the weekly treated (W-T) ones. A $27 \%$ increase in cortisol values of D-T P group was observed compared to the W-T group $(\mathrm{p}=0.101)$.

A difference both in TAC and cortisol levels of male and female patients was also observed. Lower antioxidant levels $(21 \%$ decrease, $\mathrm{p}=0.055)$ and higher cortisol levels of poor significance though (29\% increase, $\mathrm{p}=$ 0.214 ), were measured in male patients compared to the female ones as shown in Figure 3.

\section{DISCUSSION}

Low antioxidant capacity was accompanied by high cortisol levels in cancer patients on chemotherapy, although some studies showed that cortisol can decrease

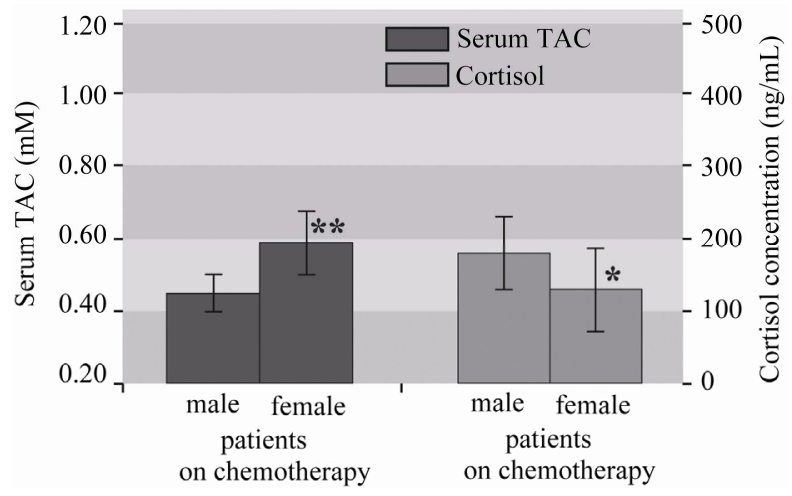

Figure 3. Total antioxidant capacity and cortisol levels in blood serum of male $(n=18)$ and female $(n=33)$ cancer patients receiving chemotherapy (significance levels according to the Student's t-test: $\left.{ }^{*} \mathrm{p}=0.214,{ }^{* *} \mathrm{p}=0.055\right)$. The vertical bars represent SD. 
oxidative stress through inhibition of nuclear factor- $\kappa \mathrm{B}$ $[37,38]$ The decrease in antioxidant capacity reached $72 \%$ in weekly treated patients and $82 \%$ in daily treated patients compared to the control group. Since, most of the chemotherapeutic agents are cleared-off within hours or a few days, this means that a slight part of the decrease observed was due to the presence of the chemotherapeutic agent itself and low TAC was observed at least seven days after application of chemotherapy. This was a common finding in all patients and was not dependent on the chemotherapeutic agent used. A $10 \%$ $20 \%$ decrease in TAC is a common characteristic in patients suffering from cancer and was observed in benign and malignant neoplasias [39]. Emotional and biological stress and cytotoxic procedures may also contribute to that decrease. Increased cortisol concentrations were measured in all patients. A $10 \%$ average increase was observed in weekly-treated patients reaching 64\% increase in daily-treated patients. Although, a straight relationship between antioxidant status and cortisol levels was not obtained from our results, high cortisol concentration may partly contribute to TAC decrease especially in daily-treated patients.

Exposure to stressful conditions often leads to high cortisol levels, smoothening at the same time, the differences existing because of sex or age [40]. Some published studies showed a significant reduction in serum cortisol immediately after infusion of either cisplatin or carboplatin and other chemotherapeutic agent like lysodren which works by attacking the cells of the adrenal glands to inhibit adrenal gland functioning [41].

However, this was not observed in the present study. It seems that additional excretion of the hormone due to chemotherapy is added to the already elevated levels in male patients [42] or that male patients respond in a different way in stressful conditions. Different response to chemotherapy is also reflected in the antioxidant levels in serum of the two sexes. Sex differences in symptoms and response to a therapy has often been observed in cancer patients [43].

In spite of the suggestion of some researchers that treatment with antioxidants may reduce the effect of some anticancer agents, we believe that such a great and persistent decrease in antioxidant capacity accompanying increased cortisol concentrations augments the probability of harmful side-effects and may lead to the organism weakening. We conclude that, depending on the treatments used against cancer, a special diet or treatment aiming at the improvement of patients' antioxidant capacity and reduction of cortisol concentration may upgrade the health status of patients and their response to therapies in general.

\section{ACKNOWLEDGEMENTS}

The authors thank Dr Yannis Kazoglou for useful comments on the manuscript.

\section{REFERENCES}

[1] Skrzydlewska, E., Stankiewicz, A., Sulkowska, M., Sulkowski, S. and Kasacka, I. (2001) Aantioxidant status and lipid peroxidation in colorectal cancer. Journal of Toxicology and Environmental Health, 64, 213-222. doi:10.1080/15287390152543690

[2] Shacter, E., Williams, J.A., Hinson, R.M., Senturker, S. and Lee, Y.J. (2000) Oxidative stress interferes with cancer chemotherapy: Inhibition of lymphoma cell apoptosis and phagocytosis. Blood, 96, 307-313.

[3] Lee, Y.J. and Shacter, E. (1999) Oxidative stress inhibits apoptosis in human lymphoma cells. Journal of Biological Chemistry, 274, 19792-19798.

doi:10.1074/jbc.274.28.19792

[4] Weijl, N.I., Leton, F.J. and Osanto, S. (1997) Free radicals and antioxidants in chemotherapy-induced toxicity. Cancer Treatment Reviews, 23, 209-240. doi:10.1016/S0305-7372(97)90012-8

[5] Durken, M., Agbenu, J., Finckh, B., Hübner, C., Pichlmeier, U., Zeller, W., et al. (1995) Deteriorating free radicaltrapping capacity and antioxidant status in plasma during bone marrow transplantation. Bone Marrow Transplant, 15, 757-762.

[6] Lauterburg, B.H., Nguyen, T., Hartmann, B., Junker, E., Kupfer, A. and Cerny, T. (1994) Depletion of total cysteine, glutathione, and homocysteine in plasma by ifosfamide/mesna therapy. Cancer Chemotherapy and Pharmacology, 35,132-136. doi:10.1007/BF00686635

[7] Crohns, M., Liippo, K., Erhola, M., Kankaanranta, H., Moilanen, E., Alho, H., et al. (2009) Concurrent decline of several antioxidants and markers of oxidative stress during combination chemotherapy for small cell lung cancer. Clinical Biochemistry, 42, 1236-1245.

doi:10.1016/j.clinbiochem.2009.05.003

[8] Ladner, C., Ehninger, G., Gey, K.F. and Clemens, M.R. (1989) Effect of etoposide (VP16-213) on lipid peroxidation and antioxidant status in a highdose radiochemotherapy regimen. Cancer Chemotherapy and Pharmacology, 25, 210-212. doi:10.1007/BF00689585

[9] Faure, H., Coudray, C., Mousseau, M., Ducros, V., Douki, T., Bianchini, F., et al. (1996) 5-Hydroxymethyluracil excretion, plasma TBARS and plasma antioxidant vitamins in adriamycin-treated patients. Free Radical Biology and Medicine, 20, 979-983.

doi:10.1016/0891-5849(95)02187-6

[10] Bhuvarahamurthy, V., Balasubramanian, N. and Govindasamy, S. (1996) Effect of radiotherapy and chemotherapy on circulating antioxidant system of human uterine cervical carcinoma. Molecular and Cellular Biochemistry, 158, 17-23.

[11] Gadjeva, V., Dimov, A. and Georgieva, N. (2008) Influence of therapy on the antioxidant status in patients with melanoma. Journal of Clinical Pharmacy and Therapeutics, 33, 179-185. doi:10.1111/j.1365-2710.2008.00909.x

[12] Papageorgiou, M., Stiakaki, E., Dimitriou, H., Malliaraki, N., Notas, G., Castanas, E., et al. (2005) Cancer chemotherapy reduces plasma total antioxidant capacity in children with malignancies. Leukemia Research, 29, 11- 
16. doi:10.1016/j.leukres.2004.04.017

[13] Ten Kate, M., van der Wal, J.B., Sluiter, W., Hofland, L.J., Jeekel, J., Sonneveld, P., et al. (2008) The role of superoxide anions in the development of distant tumour recurrence. British Journal of Cancer, 98, 580-586.

[14] Burstein, H.J., Gelber, S., Guadagnoli, E. and Weeks, J.C. (1999) Use of alternative medicine by women with early-stage breast cancer. New England Journal of Medicine, 340, 1733-1739. doi:10.1056/NEJM199906033402206

[15] Van de Creek, L., Rogers, E. and Lester, J. (1999) Use of alternative therapies among breast cancer outpatients compared with the general population. Alternative Therapies in Health and Medicine, 5, 71-76.

[16] D'Andrea, G.M. (2005) Use of antioxidants during chemotherapy and radiotherapy should be avoided. A Cancer Journal for Clinicians, 55, 319-321.

[17] Pace, A., Savarese, A., Picardo, M., Maresca, V., Pacetti, U., Del Monte, G., et al. (2003) Neuroprotective effect of vitamin E supplementation in patients treated with cisplatin chemotherapy. Journal of Clinical Oncology, 21, 927-931. doi:10.1200/JCO.2003.05.139

[18] Iarussi, D., Auricchio, U., Agretto, A., Murano, A., Giuliano, M., Casale, F., et al. (1994) Protective effect of coenzyme Q10 on anthracyclines cardiotoxicity: Control study in children with acute lymphoblastic leukemia and non-Hodgkin lymphoma. Molecular Aspects of Medicine, 15, 207-212.

[19] Weijl, N.I., Elsendoorn, T.J., Lentjes, E.G., Hopman, G.D., Wipkink-Bakker, A., Zwinderman, A.H., et al. (2004) Supplementation with antioxidant micronutrients and chemotherapy-induced toxicity in cancer patients treated with cisplatin-based chemotherapy: A randomised, double-blind, placebo-controlled study. European Journal of Cancer, 40, 1713-1723.

[20] Guaiquil, V.H., Vera, J.C. and Golde, DW. (2001) Mechanism of vitamin $\mathrm{C}$ inhibition of cell death induced by oxidative stress in glutathione-depleted HL-60 cells. Journal of Biological Chemistry, 276, 40955-40961. doi:10.1074/jbc.M106878200

[21] Lesperance, M.L., Olivotto, I.A., Forde, N., Zhao, Y., Speers, C., Foster, H., et al. (2002) Mega-dose vitamins and minerals in the treatment of non-metastatic breast cancer: An historical cohort study. Breast Cancer Research and Treatment, 76, 137-143. doi:10.1023/A:1020552501345

[22] Bjelakovic, G., Nikolova, D., Simonetti, R.G. and Gluud, C. (2004) Antioxidant supplements for prevention of gastrointestinal cancers: A systematic review and metaanalysis. Lancet, 364, 1219-1228.

[23] Greggi Antunes, L.M., Darin, J.D. and Bianchi, M.D. (2000) Protective effects of vitamin C against cisplatininduced nephrotoxicity and lipid peroxidation in adult rats: A dose-dependent study. Pharmacological Research, 41, 405-411. doi:10.1006/phrs. 1999.0600

[24] Chinery, R., Brockman, J.A., Peeler, M.O., Shyr, Y., Beauchamp, R.D. and Coffey, R.J. (1997) Antioxidants enhance the cytotoxicity of chemotherapeutic agents in colorectal cancer: A p53-independent induction of p21 WAF1/CIP1 via C/EBPbeta. Nature Medicine, 3, 12331241.

[25] Daubeuf, S., Balin, D., Leroy, P. and Visvikis, A. (2003)
Different mechanisms for gamma-glutamyltranspeptidase-dependent resistance to carboplatin and cisplatin. Biochemical Pharmacology, 66, 595-604.

[26] Agelaki, S., Tsatsanis, C., Gravanis, A. and Margioris, A.N. (2002) Corticotropin-releasing hormone augments proinflammatory cytokine production from macrophages in vitro and in lipopolysaccharide-induced endotoxin shock in. Infection and Immunity, 70, 6068-6074.

[27] Lovallo, W.R., Farag, N.H., Vincent, A.S., Thomas, T.L. and Wilson, M.F. (2006) Cortisol responses to mental stress, exercise, and meals following caffeine intake in men and women. Pharmacology Biochemistry and Behaviour, 83, 441-447. doi:10.1016/j.pbb.2006.03.005

[28] Ruzic, B., Tomaskovic, I., Trnski, D., Kraus, O., Bekavac-Beslin, M. and Vrkic, N. (2005) Systemic stress responses in patients undergoing surgery for benign prostatic hyperplasia. BJU International, 95, 77-80.

[29] Lutgendorf, SK., Weinrib, A.Z., Penedo, F., Russell, D., DeGeest, K. and Costanzo, E. (2008) Interleukin-6, cortisol, and depressive symptoms in ovarian cancer patients. Journal of Clinical Oncology, 26, 4820-4827. doi:10.1200/JCO.2007.14.1978

[30] Young, A.H. (2004) Cortisol in mood disorders. Stress, 7, 205-208.

[31] Wang, L., Muxin, G., Nishida, H., Shirakawa, C., Sato, S. and Konishi, T. (2007) Psychological stress-induced oxidative stress as a model of sub-healthy condition and the effect of TCM. Evidence Based Complementary and Alternative Medicine, 4, 195-202.

[32] Marusin, A.V., Salyukov, V.B. and Yu Bragina, E. (2002) Antioxidant activity of blood plasma in individuals with neoplasms. Bulletin of Experimental Biology and Medicine, 133, 481-483.

[33] Thakor, A.S. and Giussani, D.A. (2005) Antioxidants enhance the adrenocortical response to stress in the fetus. Endocrine Abstracts, 196th Meeting of the Society for Endocrinology, London, UK, 7-9 November 2005, 10 OC22

[34] Erel, O.A. (2004) Novel automated method to measure total antioxidant response against potent free radical reactions. Clinical Biochemistry, 37, 112-119.

[35] Perry, L.A. and Gossman, A.B. (1997) The role of the laboratory in the diagnosis of Cushing's syndrome. Annals of Clinical Biochemistry, 34, 345-359.

[36] Press, W.H., Teukolsky, S.A., Vetterling, W.T. and Flannery B.P. (1992) Numerical Recipes in C: The art of scientific computing. Cambridge University Press, Cambridge.

[37] Almawi, W.Y. and Melemedjian, O.K. (2002) Negative regulation of nuclear factor-kappab activation and function by glucocorticoids. Journal of Molecular Endocrinology, 28, 69-78. doi:10.1677/jme.0.0280069

[38] Marumo, T., Schini-Kerth, V.B., Brandes, R.P. and Busse, R. (1998) Glucocorticoids inhibit superoxide anion production and p22 phox mRNA expression in human aortic smooth muscle cells. Hypertension, 32, 1083-1088.

[39] Pejić, S., Todorović, A., Stojiljković, V., Cvetković, D., Lucić, N., Radojicić, R.M., et al. (2008) Superoxide dismutase and lipid hydroperoxides in blood and endometrial tissue of patients with benign, hyperplastic and malignant endometrium. Annals of the Brazilian Academy of Science, 80, 515-522. 
[40] Swaab, D.F., Raadsheer, F.C., Endert, E., Hofman Kamphorst, M.W. and Ravid, R. (2006) Increased cortisol levels in Aging and Alzheimer's disease in postmortem cerebrospinal fluid. Journal of Neuroendocrinology, 6, 681-687. doi:10.1111/j.1365-2826.1994.tb00635.x

[41] Morrow, G.R., Hickok, J.T., Andrews, P.L. and Stern, R.M. (2002) Reduction in serum cortisol after platinum based chemotherapy for cancer: A role for the HPA axis in treatment-related nausea? Psychophysiology, 39, 491495. doi:10.1111/1469-8986.3940491

[42] Paris, J.J., Franco, C., Sodano, R., Freidenberg, B.,
Gordis, E., Anderson, D.A., et al. (2010) Sex differences in salivary cortisol in response to acute stressors among healthy participants, in recreational or pathological gamblers, and in those with posttraumatic stress disorder. Hormones and Behaviour, 57, 35-45. doi:10.1016/j.yhbeh.2009.06.003

[43] McCann, J. (2000) Gender differences in cancer that don't make sense-Or do they? Journal of the National Cancer Institute, 92, 1560-1562. doi:10.1093/jnci/92.19.1560 